\title{
Probabilistic Scan Matching for Motion Estimation in Unstructured Environments
}

\author{
Luis Montesano Javier Minguez Luis Montano \\ Instituto de Investigación en Ingeniería de Aragón \\ Departamento de Informática e Ingeniería de Sistemas, Universidad de Zaragoza, Spain \\ \{montesano,jminguez,montano\}@unizar.es
}

\begin{abstract}
This paper presents a probabilistic scan matching algorithm to estimate the robot planar displacement by matching dense two-dimensional range scans. The general framework follows an iterative process of two steps: $(i)$ computation of correspondences between scans, and (ii) estimation of the relative displacement. The contribution is a probabilistic modelling of this process that takes into account all the uncertainties involved: the uncertainty of the displacement of the sensor and the measurement noises. Furthermore, it also considers all the possible correspondences resulting from these uncertainties. This technique has been implemented and tested on a real vehicle. The experiments illustrate how the performances of this method are better than previous geometric ones in terms of robustness, accuracy and convergence.
\end{abstract}

\section{INTRODUCTION}

In this paper we present a new probabilistic scan matching algorithm. The objective of the scan matching techniques is to compute the relative motion of the sensor between two consecutive configurations by maximizing the overlap between the sensor measurements obtained at each configuration. These techniques have been widely used in the context of mapping, 3D data registration, object recognition or scene understanding [18], [12]. In robotics, they have been successfully used in a wide range of applications as an ameliorated odometry (i.e. simultaneous location and map building [20], [8], [10], local map construction [14], [11], [15] or people tracking systems [19]).

One of the main differences between the existing algorithms is the usage or not of high-level entities such as lines or planes. In structured environments, one can assume the existence of polygonal structure in the environment [7], [5]. These methods are fast and work quite well in indoor environments. However, they limit the scope of application to the extraction of geometric features that are not always available in unstructured environments. On the other hand, a great deal of work has been done to perform in any type of scenario dealing with raw data. For example [3] constructs a piecewise continuous differentiable density that models on a grid the probability to measure a point, and then, apply the Newton's algorithm to estimate the relative motion. By converting the scans to statistical representations, [21] iteratively computes the crosscorrelation that results in the displacement. In [6] the motion parameters are estimated using a constrained velocity equation for the scanned points. Our working context are these unstructured scenarios.

The most popular methods usually follow the Iterative Closest Point (ICP) algorithm [2]. They are based on an iterative process where they first compute the correspondences between the scans, and then they minimize the distance error to compute the sensor displacement [13], [9], [1], [17]. This process is repeated with this new estimate until convergence. A common feature of most versions of ICP is the usage of the Euclidean distance to establish the correspondences and to apply the least squares minimization. As pointed out by [13], the limitation of this distance is to capture the rotation of the sensor. For instance, points far from the sensor could be far from its correspondent due to the rotation error of the sensor. Moreover, the correspondences might not capture the correct motion between the scans. We understand that this is a central problem of the ICP algorithms: to find a measure that captures the sensor translation and rotation at the same time. To overcome this limitation [13] proposed to combine the closest point rule of the ICP algorithm with a range rule to capture the sensor rotation. This method performs well under large rotation errors. However, it is a geometric algorithm that do not model the uncertainties involved in the process.

In fact, another important difference of the scan matching techniques is whether they model the noises associated to the sensor measurement process and the uncertainty of the displacement between both scans. We understand that this is another central problem in the sensor matching techniques since the sensory process is uncertain, and it might be taken into account in the scan matching context. This issue has been partially addressed in [17] modelling the noise of the sensor measurements. However, they do not include the uncertainty of the sensor location. Furthermore, another remaining issue is to consider all the possible associations resulting from these uncertainties.

This paper presents a probabilistic formulation of the scan matching problem. The general framework follows an iterative process of two steps: $(i)$ probabilistic computation of the correspondences between the scans, and (ii) estimation of the relative displacement. With this formulation we address the two problems of the scan matching algorithms mentioned above. First we take into account the uncertainties of the displacement of the sensor and the observation noises to establish the correspondences. 


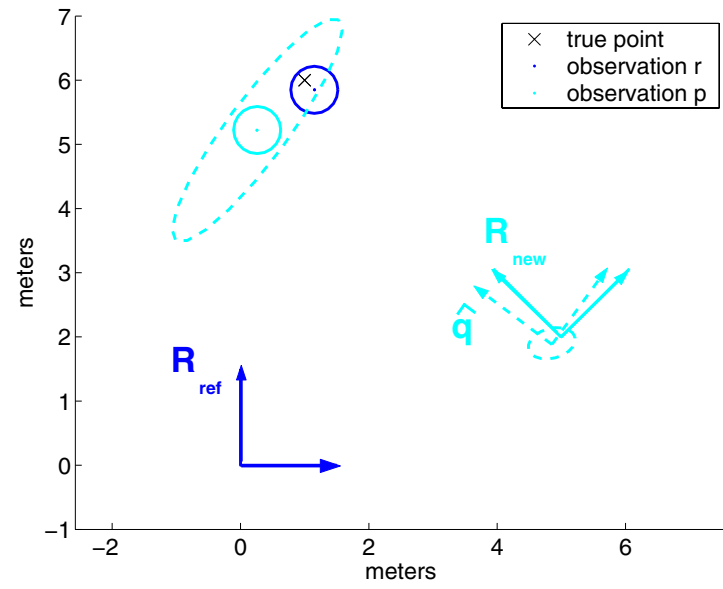

Fig. 1. Probabilistic model. The figure shows the representation of the relative displacement $\mathbf{q}$ and the points $\mathbf{p}$ and $\mathbf{r}$. The cross represents the true physical point, the dark dot is the observation acquired at $\mathbf{R}_{\text {new }}$ and the light one is the position of the observation acquired in $\mathbf{R}_{\text {ref }}$ according to the estimate of the relative pose $\hat{\mathbf{q}}$. The continuous circles represent the measurement noises of each observation. Taking into account the uncertainty of the relative displacement $\mathbf{q}$ makes the observation $\mathbf{r}$ and $\mathbf{p}$ compatibles (light dotted ellipse).

Moreover, our probabilistic model allows to estimate these correspondences integrating over all the potential associations between the points of each scan. Second, the translation and rotation are implicitly captured in this framework simultaneously. With this new method, we obtain results that ameliorate the algorithm that we were using [13] in terms of robustness, convergence and precision.

The paper is organized as follows: Section II introduces the probabilistic model. Section III presents the scan matching algorithm. In Section IV we discuss the experimental results and compare the method with existing methods. Finally, the conclusions are presented in Section $\mathrm{V}$.

\section{Probabilistic Model and Notation}

Let $S_{\text {ref }}=\left\{\mathbf{r}_{1}, \ldots, \mathbf{r}_{\mathbf{n}}\right\}$ be a set of $n$ point measurements $\left(r_{\mathbf{i}} \in \mathbf{R}^{2}\right)$ acquired in a given sensor location $R_{\text {ref }}$, and $S_{\text {new }}=\left\{\mathbf{p}_{\mathbf{1}}, \ldots, \mathbf{p}_{\mathbf{m}}\right\}$ another set of $m$ point measurements acquired in another sensor location $R_{\text {new }}$. Let $\mathbf{q}=\{x, y, \theta\} \in \mathbf{R}^{2} \times[0,2 \pi]$ be the relative location of $R_{\text {new }}$ with respect to $R_{\text {ref }}$. The problem is to estimate the sensor displacement $\mathbf{q}$ such that maximizes the overlap between $S_{\text {ref }}$ and $S_{\text {new }}$.

We model the scan matching problem within a probabilistic framework where the observed points $\mathbf{r}_{\mathbf{i}}$ and $\mathbf{p}_{\mathbf{i}}$ and the relative displacement $\mathbf{q}$ are random variables. Our model considers the first two moments of the distributions of these random variables. We consider that the observations of the points in both scans are independent and corrupted with white zero mean noises with covariance matrices $\mathbf{P}_{\mathbf{r}_{\mathbf{i}}}$ and $\mathbf{P}_{\mathbf{p}_{\mathbf{i}}}{ }^{1}$,

\footnotetext{
${ }^{1}$ Without loss of generality in the following we assume that the points are given in Cartesian coordinates. In the practical case of a laser range sensor $\mathbf{p}_{i}=\left\{\hat{\mathbf{p}}_{i}, \mathbf{P}_{\mathbf{p}_{\mathbf{i}}}\right\}$ are computed from the polar coordinates and the sensor uncertainty parameters using the Jacobian of the transformation between polar and Cartesian coordinates.
}

$$
\begin{aligned}
& \hat{\mathbf{r}}_{\mathbf{i}}=\mathbf{r}_{i}^{\text {true }}+w_{r_{i}}, \quad w_{r_{i}} \sim N\left(0, \mathbf{P}_{\mathbf{r}_{\mathbf{i}}}\right) \\
& \hat{\mathbf{p}}_{\mathbf{i}}=\mathbf{p}_{i}^{\text {true }}+w_{p_{i}}, \quad w_{p_{i}} \sim N\left(0, \mathbf{P}_{\mathbf{p}_{\mathbf{i}}}\right)
\end{aligned}
$$

where $\hat{\mathbf{r}}_{\mathbf{i}}$ and $\hat{\mathbf{p}}_{\mathbf{i}}$ represent the observations of the true physical points $\mathbf{p}_{i}^{\text {true }}$ and $\mathbf{r}_{i}^{\text {true }}$. Let $\hat{\mathbf{q}}$ be the estimation of the relative location. The initial displacement $\hat{\mathbf{q}}_{0}$ and its associated covariance matrix $\mathbf{P}_{\mathbf{q}}$ represent the prior knowledge about the relative location of $R_{\text {new }}$ with respect to $R_{\text {ref }}$. Figure 1 illustrates our probabilistic model and how the error on the relative location affects the position of the points in $S_{\text {new }}$.

\section{Probabilistic SCAN Matching}

The objective is to estimate the relative displacement $\mathbf{q}$. A common approach is to perform an iterative process in two steps [2]. At each iteration $k$, the algorithm establishes for each point $\mathbf{p}_{\mathbf{i}}$ in $S_{\text {new }}$ a correspondence $\mathbf{a}_{\mathbf{i}}$ using the current estimate $\hat{\mathbf{q}}_{k}$ and the points in $S_{r e f}$. The result is a set of pairs of points $\left\langle\mathbf{a}_{\mathbf{i}}, \mathbf{p}_{\mathbf{i}}\right\rangle$. Next, a new estimation of the relative location $\hat{\mathbf{q}}_{\mathbf{k}+\mathbf{1}}$ is computed by minimizing the error of these correspondences.

The problem of establishing the correspondences between scans is crucial in this process. This is difficult when using raw data in the presence of large errors in the sensor displacement estimation and noises on the observations. Moreover, sensors usually have a discrete angular resolution which implies that the observations do not necessarily correspond to the same physical points.

We describe next both steps with an special emphasis in the correspondences, since it is a key point of this technique.

\section{A. Computing the correspondences (Step 1)}

The objective is to compute for each $\mathbf{p}_{\mathbf{i}}$ of $S_{\text {new }}$ a correspondence $\mathbf{a}_{\mathbf{i}}$ from the set of reference points $S_{r e f}$. We address this by reducing the initial set of all possible correspondences $S_{\text {ref }}$ to a subset $A_{i}$ that contains all the points in $S_{r e f}$ statistically compatible with $\mathbf{p}_{\mathbf{i}}$. Next, we compute the correspondence $\mathbf{a}_{\mathbf{i}}$ by integrating over all the potential associations of $A_{i}$.

To compute the compatible associations of $S_{\text {ref }}$, we perform an individual compatibility test based on the Mahalanobis distance $D_{M}\left(\mathbf{p}_{\mathbf{i}}, \mathbf{r}_{\mathbf{j}}\right)$ between $\mathbf{p}_{\mathbf{i}}$ and $\mathbf{r}_{\mathbf{j}}$,

$$
D_{M}^{2}\left(\mathbf{p}_{\mathbf{i}}, \mathbf{r}_{\mathbf{j}}\right)=\delta_{i j} \mathbf{C}_{\mathbf{i j}}{ }^{-1} \delta_{i j}
$$

where $\delta_{i j}=\mathbf{f}\left(\mathbf{q}_{k}, \mathbf{p}_{\mathbf{i}}\right)-\mathbf{r}_{\mathbf{j}}$. The function $\mathbf{f}$ transforms the point $\mathbf{p}_{\mathbf{i}}$ to the reference system $R_{\text {ref }}$ through the relative location $\mathbf{q}$,

$$
\mathbf{f}(\mathbf{q}, \mathbf{p})=\left(\begin{array}{c}
\cos \theta p_{x}-\sin \theta p_{y}+x \\
\sin \theta p_{x}+\cos \theta p_{y}+y
\end{array}\right)
$$

Linearizing $\mathbf{f}$ around the current estimate $\hat{\mathbf{q}}_{k}$ and the observed point $\hat{\mathbf{p}}_{\mathbf{i}}$ using a first order Taylor approximation, the matrix $\mathbf{C}_{\mathbf{i j}}$ has the following expression, 


$$
\mathbf{C}_{\mathbf{i j}}=\mathbf{P}_{\mathbf{r}_{\mathbf{j}}}+\mathbf{J}_{\mathbf{q}}\left(\hat{\mathbf{q}}_{k}, \hat{\mathbf{p}}_{\mathbf{i}}\right) \mathbf{P}_{\mathbf{q}} \mathbf{J}_{\mathbf{q}}\left(\hat{\mathbf{q}}_{k}, \hat{\mathbf{p}}_{\mathbf{i}}\right)^{T}+\mathbf{J}_{\mathbf{p}}\left(\hat{\mathbf{q}}_{k}\right) \mathbf{P}_{\mathbf{p}_{\mathbf{i}}} \mathbf{J}_{\mathbf{p}}\left(\hat{\mathbf{q}}_{k}\right)^{T}
$$

The matrices $\mathbf{J}_{\mathbf{q}}(\hat{\mathbf{q}}, \hat{\mathbf{p}})$ and $\mathbf{J}_{\mathbf{p}}(\hat{\mathbf{q}})$ are the Jacobians of the function $\mathbf{f}$ with respect to $\mathbf{q}$ and $\mathbf{p}_{\mathbf{i}}$ evaluated at the current estimate $\hat{\mathbf{q}}_{k}$ and the point $\hat{\mathbf{p}}_{\mathbf{i}}$,

$$
\mathbf{J}_{\mathbf{q}}\left(\hat{\mathbf{q}}_{k}, \hat{\mathbf{p}}_{\mathbf{i}}\right)=\left.\frac{\partial \mathbf{f}(\mathbf{q}, \mathbf{r}, \mathbf{p})}{\partial \mathbf{q}}\right|_{\hat{\mathbf{q}}_{k}, \hat{\mathbf{p}}_{\mathbf{i}}} \mathbf{J}_{\mathbf{p}}\left(\hat{\mathbf{q}}_{k}\right)=\left.\frac{\partial \mathbf{f}(\mathbf{q}, \mathbf{r}, \mathbf{p})}{\partial \mathbf{p}}\right|_{\hat{\mathbf{q}}_{k}}
$$

Under Gaussian assumptions the Mahalanobis distance has a chi-squared distribution with $m=\operatorname{dim}\left(\delta_{i j}\right)$ degrees of freedom. A point $\mathbf{r}_{\mathbf{j}}$ is compatible iff $D_{M}^{2}\left(\mathbf{p}_{\mathbf{i}}, \mathbf{r}_{\mathbf{j}}\right)<$ $\chi_{d, \alpha}^{2}$, where $\alpha$ is the desired confidence level of the chisquared test. Using this procedure for all points of $S_{r e f}$ we get the set $A_{i}=\left\{\mathbf{a}_{\mathbf{i} 1}, \ldots, \mathbf{a}_{\mathbf{i k}}\right\}$ of compatible associations for a given $\mathbf{p}_{\mathbf{i}}$.

We model $\mathbf{a}_{\mathbf{i}}$ as a random variable defined over the set $A_{i}$. The expectation $\hat{\mathbf{a}}_{\mathbf{i}}$ and the covariance matrix $\mathbf{P}_{\mathbf{a}_{\mathbf{i}}}$ of $\mathbf{a}_{\mathbf{i}}$ are:

$$
\begin{aligned}
\hat{\mathbf{a}}_{\mathbf{i}} & =E\left[\mathbf{a}_{\mathbf{i}}\right]=\sum_{j} \mathbf{a}_{\mathbf{i j}} p\left(\mathbf{a}_{\mathbf{i j}}\right) \\
\mathbf{P}_{\mathbf{a}_{\mathbf{i}}} & =\sum_{j}\left(\mathbf{a}_{\mathbf{i j}}-\hat{\mathbf{a}}_{\mathbf{i}}\right)\left(\mathbf{a}_{\mathbf{i} \mathbf{j}}-\hat{\mathbf{a}}_{\mathbf{i}}\right)^{T} p\left(\mathbf{a}_{\mathbf{i j}}\right)
\end{aligned}
$$

The term $p\left(\mathbf{a}_{\mathbf{i j}}\right)$ represents the probability of point $\mathbf{r}_{\mathbf{j}}$ being the correspondent point of $\mathbf{p}_{\mathbf{i}}$. In order to take into account the uncertainty of the sensor location and the noises of the observations we integrate over all the possible locations of the point $\mathbf{p}_{\mathbf{i}}$ and all the possible locations of the sensor $\mathbf{q}$,

$$
p\left(\mathbf{a}_{\mathbf{i j}}\right)=\eta_{i} \iint \underbrace{p\left(\hat{\mathbf{r}}_{\mathbf{j}} \mid \mathbf{q}_{k}, \mathbf{p}_{\mathbf{i}}\right)}_{\sim N\left(\mathbf{f}\left(\hat{\mathbf{q}}_{k}, \hat{\mathbf{p}}_{\mathbf{i}}\right), \mathbf{P}_{\mathbf{r}_{\mathbf{j}}}\right)} \underbrace{p\left(\mathbf{p}_{\mathbf{i}}\right)}_{\sim N\left(\hat{\mathbf{p}}_{\mathbf{i}}, \mathbf{P}_{\mathbf{p}_{\mathbf{i}}}\right) \sim N\left(\hat{\mathbf{q}}_{k}, \mathbf{P}_{\mathbf{q}}\right)} \underbrace{p\left(\mathbf{q}_{k}\right)}_{\mathbf{q}_{k} d \mathbf{p}_{\mathbf{i}}}
$$

where $\eta_{i}=\sum_{j} p\left(\mathbf{a}_{\mathbf{i j}}\right)$ is a normalization coefficient assuring that the sum of the probabilities of all the correspondences of $\mathbf{p}_{\mathbf{i}}$ is one. According to the model presented in Section II $p\left(\mathbf{p}_{\mathbf{i}}\right)$ and $p\left(\mathbf{q}_{k}\right)$ are Gaussian distributions $N\left(\hat{\mathbf{p}}_{\mathbf{i}}, \mathbf{P}_{\mathbf{p}_{\mathbf{i}}}\right)$ and $N\left(\hat{\mathbf{q}}_{k}, \mathbf{P}_{\mathbf{q}}\right)$ respectively. To obtain an analytical solution for the previous equation we model $p\left(\hat{\mathbf{r}}_{\mathbf{j}} \mid \mathbf{q}_{k}, \mathbf{p}_{\mathbf{i}}\right)$ as a Gaussian distribution $N\left(\mathbf{f}\left(\hat{\mathbf{q}}_{k}, \hat{\mathbf{p}}_{\mathbf{i}}\right), \mathbf{P}_{\mathbf{r}_{\mathbf{j}}}\right)$. The resulting distribution is also Gaussian with mean $\mathbf{f}\left(\hat{\mathbf{q}}_{k}, \hat{\mathbf{p}}_{\mathbf{i}}\right)$ and covariance matrix $\mathbf{C}_{\mathbf{i j}}$. The probability $p\left(\mathbf{a}_{\mathbf{i j}}\right)$ is obtained evaluating this Gaussian at $\mathbf{a}_{\mathrm{ij}}$. Figure 2 illustrates the previous procedure.

The estimated correspondences have two important characteristics. First, since the algorithm integrates over all the possible associations, it captures the uncertainty of the correspondences. This is reflected in the covariance matrix which depends on the uncertainties of the sensor relative location, on the measurement noises and on the environment. Notice how the estimated covariance of the correspondent point of Figure 2 represents the uncertainty of the associations. Second, the use of the probabilistic

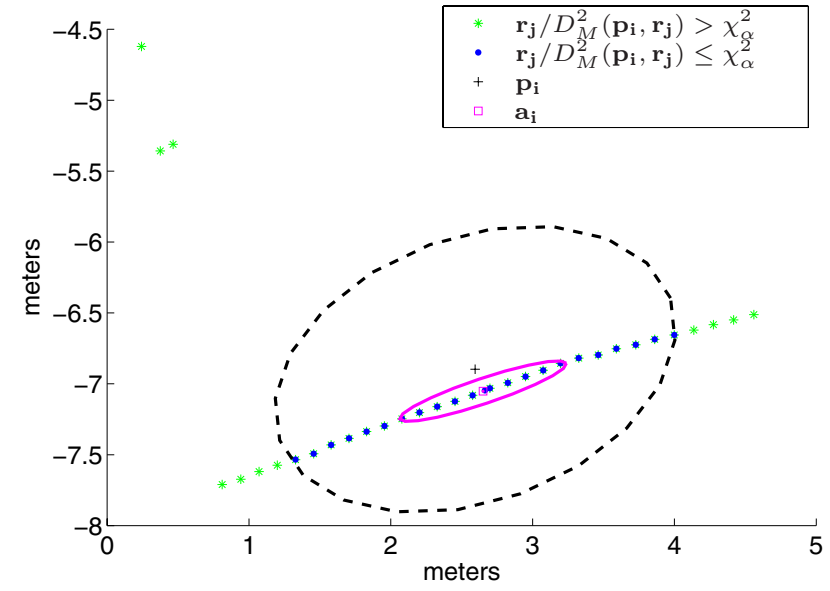

Fig. 2. Computation of the correspondent point of $\mathbf{p}_{\mathbf{i}}$ (cross). The set of possible correspondences are the points satisfying the $\chi^{2}$ test (dark points inside the dashed ellipse). The resulting mean (square point) and covariance (solid ellipse) of the correspondence depend on the measurement noises, the uncertainty of $\mathbf{q}$ and the structure of the environment.

model takes into account both the translational and the rotational error of the sensor location simultaneously (Figure $1)$.

Using the previous procedure for each point $\mathbf{p}_{\mathbf{i}}$ in $S_{\text {new }}$ we obtain a set of $t$ correspondences $S_{a}=\left\{\left\langle\mathbf{a}_{\mathbf{1}}, \mathbf{p}_{\mathbf{1}}\right\rangle\right.$ $\left., \ldots,<\mathbf{a}_{\mathbf{t}}, \mathbf{p}_{\mathbf{t}}>\right\}$. Note that $t \leq m$ since the set $A_{i}$ could be empty for some of the points in $S_{\text {new }}$.

\section{B. Estimation of relative location (Step 2)}

The objective is to improve the current estimation $\hat{\mathbf{q}}_{k}$ given the set of correspondences computed in the previous step. We use a Least Squares method to minimize the square error of these correspondences. Each pair of $S_{a}$, $\left.<\mathbf{a}_{i}, \mathbf{p}_{i}\right\rangle$, and the relative displacement of the sensor $\mathbf{q}$ are related through an implicit measurement function $\mathbf{e}$,

$$
\mathbf{e}\left(\mathbf{a}_{\mathbf{i}}, \mathbf{p}_{\mathbf{i}}, \mathbf{q}\right)=\mathbf{f}\left(\mathbf{q}, \mathbf{p}_{\mathbf{i}}\right)-\mathbf{a}_{\mathbf{i}}=0
$$

Linearizing the function $\mathbf{f}$ as in the previous section and rearranging terms we get a linear system for each correspondence,

$$
\mathbf{e}\left(\hat{\mathbf{a}}_{\mathbf{i}}, \hat{\mathbf{p}}_{\mathbf{i}}, \hat{\mathbf{q}}_{k}\right)=\mathbf{J}_{\mathbf{q}}\left(\hat{\mathbf{q}}_{k}, \hat{\mathbf{p}}_{\mathbf{i}}\right)\left(\hat{\mathbf{q}}_{k}-\mathbf{q}\right)
$$

The Least Square method minimizes the following criterium,

$$
M(\mathbf{q})=\sum_{i=1}^{n} \mathbf{e}\left(\hat{\mathbf{a}}_{\mathbf{i}}, \hat{\mathbf{p}}_{\mathbf{i}}, \hat{\mathbf{q}}_{k}\right)^{T} \mathbf{C}_{\mathbf{i}}^{-1} \mathbf{e}\left(\hat{\mathbf{a}}_{\mathbf{i}}, \hat{\mathbf{p}}_{\mathbf{i}}, \hat{\mathbf{q}}_{k}\right)
$$

where the covariance matrix $\mathbf{C}_{\mathbf{i}}$ is,

$$
\begin{aligned}
\mathbf{C}_{\mathbf{i}}=\mathbf{P}_{\mathbf{a}_{\mathbf{i}}}+\mathbf{J}_{\mathbf{q}}\left(\hat{\mathbf{q}}_{k}, \hat{\mathbf{p}}_{i}\right) & \mathbf{P}_{\mathbf{q}} \mathbf{J}_{\mathbf{q}}\left(\hat{\mathbf{q}}_{k}, \hat{\mathbf{p}}_{i}\right)^{T} \\
& +\mathbf{J}_{\mathbf{p}}\left(\hat{\mathbf{q}}_{k}\right) \mathbf{P}_{\mathbf{p}_{\mathbf{i}}} \mathbf{J}_{\mathbf{p}}\left(\hat{\mathbf{q}}_{k}\right)^{T}
\end{aligned}
$$

Equation (3) has an unique minimum $\hat{\mathbf{q}}_{\min }$ given by,

$$
\hat{\mathbf{q}}_{\text {min }}=\left[\mathbf{H}^{T} \mathbf{C}^{-1} \mathbf{H}\right]^{-1} \mathbf{H}^{T} \mathbf{C}^{-1} \mathbf{E}
$$



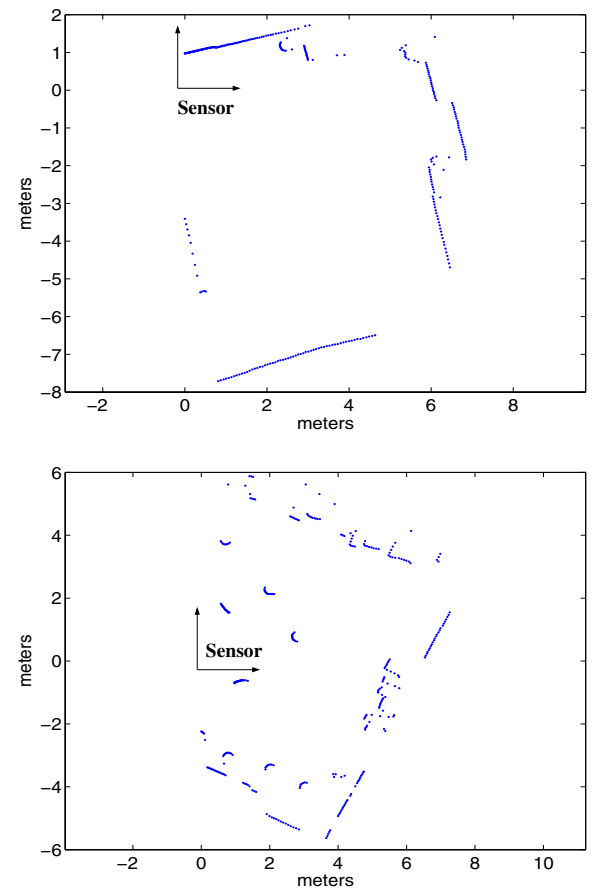

Fig. 3. Scans used in the experiment: a structured environment (top) and an unstructured one (bottom).

where $\mathbf{C}=\operatorname{diag}\left(\mathbf{C}_{\mathbf{i}}\right)$ is a block diagonal matrix of the covariance matrices $\mathbf{C}_{\mathbf{i}}$ of each correspondence and the matrices $\mathbf{H}$ and $\mathbf{E}$ are,

$$
\begin{aligned}
& \mathbf{H}=\left[\begin{array}{c}
\mathbf{J}_{\mathbf{q}}\left(\hat{\mathbf{q}}, \hat{\mathbf{p}}_{\mathbf{1}}\right) \\
\vdots \\
\mathbf{J}_{\mathbf{q}}\left(\hat{\mathbf{q}}, \hat{\mathbf{p}}_{\mathbf{t}}\right)
\end{array}\right] \\
& \mathbf{E}=\left[\begin{array}{c}
-\mathbf{e}\left(\hat{\mathbf{a}}_{\mathbf{1}}, \hat{\mathbf{p}}_{\mathbf{1}}, \hat{\mathbf{q}}_{k}\right)+\mathbf{J}_{\mathbf{q}}\left(\hat{\mathbf{q}}, \hat{\mathbf{p}}_{\mathbf{1}}\right) \hat{\mathbf{q}}_{k} \\
\vdots \\
-\mathbf{e}\left(\hat{\mathbf{a}}_{\mathbf{t}}, \hat{\mathbf{p}}_{\mathbf{t}}, \hat{\mathbf{q}}_{k}\right)+\mathbf{J}_{\mathbf{q}}\left(\hat{\mathbf{q}}, \hat{\mathbf{p}}_{\mathbf{t}}\right) \hat{\mathbf{q}}_{k}
\end{array}\right]
\end{aligned}
$$

If there is convergence the final estimate of the algorithm is $\hat{\mathrm{q}}_{\text {min }}$, if not the algorithm iterates with $\hat{\mathrm{q}}_{\mathrm{k}+\mathbf{1}}=\hat{\mathrm{q}}_{\text {min }}$.

\section{EXPERIMENTAL RESUlts}

In this Section we outline the experimental results. We tested the method with data obtained with a wheelchair mobile robot equipped with a Sick laser scanner.

To compare our method (probabilistic Iterative Correspondence, pIC in short) with existing scan matching techniques, we chose the standard ICP and the widely known IDC algorithm [13]. The IDC algorithm uses two types of correspondences (translation and rotation) and performs two minimizations. This method is designed to deal with large rotation errors.

We did not implement the IDC for the comparison. We accumulate experience in the IDC method since we have had this tool working in our laboratory for some years [16], [14], [15]. In our IDC implementation, we have modified the rotation rule to be more robust to the sensor noise and we interpolate between successive range points

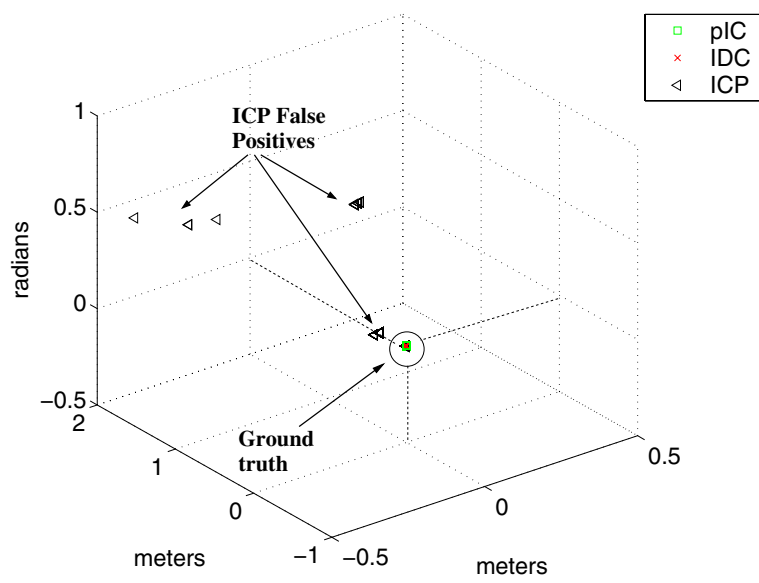

(a)

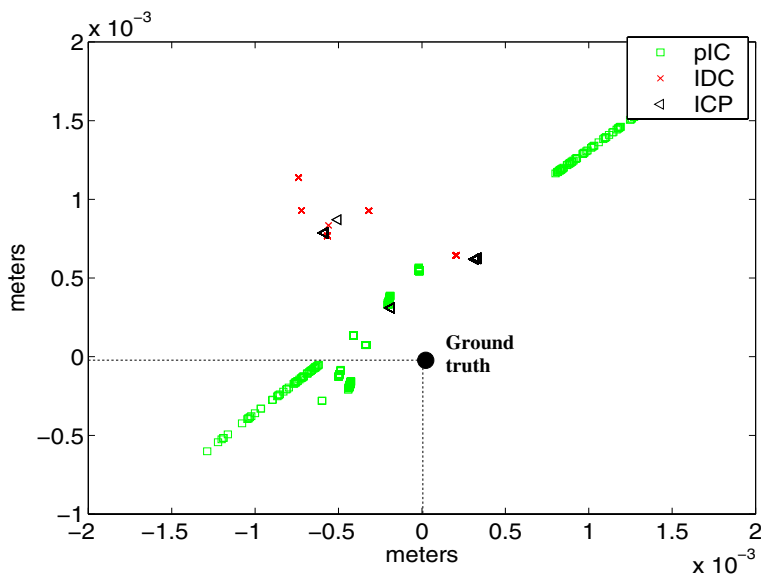

(b)

Fig. 4. (a) Estimations of the three methods. (b) Zoom on the estimations around the ground truth projected in the $X Y$ plane.

(local structure). We reject outliers using visibility criteria [13] and range criterions [17]. Furthermore, we use a trimmed version of the ICP to manage the correspondences [4] that improves the least squares minimization, and a smooth criterion of convergence [17]. We also implemented these features in the ICP algorithm. In order to show a fair comparison, we used the same values for common parameters (actually we used our IDC previous parameters for the ICP, and $\mathrm{pIC}$ ). The covariance matrix of the initial estimation for the pIC was computed from the odometry readings or set according to the noise introduced through the experiments (this is the only ad hoc parameter but with a clear physical sense).

We outline next two types of experiments. The first one evaluates the properties of the pIC algorithm by matching a pair of scans for random location errors. The second one evaluates the global algorithm performance with a run with the vehicle within our university (Figures 6).

The first experiment consisted on matching two different scans acquired in the same sensor location. Thus, the scans are different due to the sensor noise and we know precisely the ground truth $(0,0,0)$. We added random noise to the initial location estimate up to $0.2 \mathrm{~m}$ in $x$ and $y$, and up 

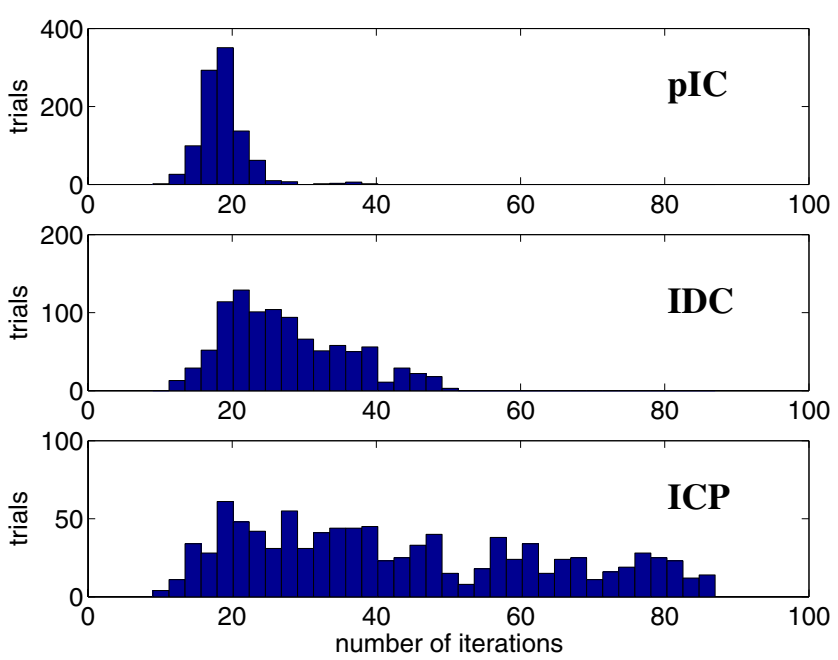

Fig. 5. Convergence rate (without the ICP negatives).

to $45^{\circ}$ in $\theta$. Notice how large are the maximum errors especially in rotation. Convergence of the algorithm was achieved when the error ratio was below $0.0001 \%$ and the maximum number of iterations was 500 . We perform this test in two different scenarios (structured and non structured, Figure 3). The scans were taken in a place where the range information was more or less equally distributed in all directions, which is a well-conditioned situation for the methods. We repeated each experiment 500 times for each scenario (a total of 1000 runs).

Figure 4a depicts the final estimates of all the methods. The pIC and IDC converged all the times and all the estimates concentrate around the true solution $(0,0,0)$. In other words, all the results of the three methods were True Positive (see Table below). On the other hand, in $1.1 \%$ of the trials the ICP did not converge leading to negatives. Another measure of robustness is that some of the ICP positives were False Positives. They corresponded to situations were the ICP converged but towards a local minimum that did not correspond to the real solution (Figure 4a).

TABLE I

PIC VS IDC AND ICP (\%)

\begin{tabular}{|c|c|c|c|c|}
\hline & Positive & False Positive & Negative & False Negative \\
\hline pIC & 100 & 0 & 0 & 0 \\
\hline IDC & 100 & 0 & 0 & 0 \\
\hline ICP & 92.9 & 6 & 1.1 & 0 \\
\hline
\end{tabular}

These results show that the pIC and the IDC are more robust than the ICP: $(i)$ all the results of the pIC and IDC were True Positives while the ICP had Negatives (they correspond to large errors in orientation that could not be compensated). And (ii) the ICP had $1.1 \%$ of False Positives (also due to large errors in orientation) which are really bad for these methods since the estimate is wrong although the result is positive. The pIC is as robust as the IDC facing large errors in rotation (our method performs as good as methods designed to deal with these situations).

Figure $4 \mathrm{~b}$ depicts a zoom on Figure $4 \mathrm{a}$ projected on the $X Y$ plane. Many solutions of the pIC concentrate closer to the ground truth than the solutions of the IDC and ICP, and some others further. They tend to concentrate in two different clusters for each scan and algorithm. Next table depicts the mean and standard deviation of the error in both coordinates (we only use the True positives):

TABLE II

PIC VS IDC AND ICP ERROR

\begin{tabular}{|c||c|c|c|c|c|c|}
\hline \multicolumn{1}{|c||}{} & \multicolumn{2}{c|}{ x error } & \multicolumn{2}{c|}{ y error } & \multicolumn{2}{c|}{ th error } \\
\cline { 2 - 7 } & $\mu$ & $\sigma$ & $\mu$ & $\sigma$ & $\mu$ & $\sigma$ \\
\hline \hline pIC $\left(10^{-3}\right)$ & 0.3 & 0.4 & 0.4 & 0.4 & $<0.0$ & $<0.0$ \\
\hline IDC $\left(10^{-3}\right)$ & 0.4 & 0.22 & 0.8 & 0.16 & $<0.0$ & $<0.0$ \\
\hline ICP $\left(10^{-3}\right)$ & 0.3 & 0.13 & 0.4 & 0.16 & $<0.0$ & $<0.0$ \\
\hline
\end{tabular}

When the algorithm converges to the right solution, the medium and covariance of the errors are very similar. The pIC seems as accurate as the IDC, but the errors are so small (sub millimeter precision) that are not significant.

Figure 5 depicts the number of iterations for each trial. The convergence rate is better in the pIC than in the IDC and ICP. The pIC converges faster than all the algorithms. This is because it deploys a correspondence process where the set of possible correspondences are used and captures the statistically possible local structure of the scenario.

We remark that this test was carried out with large errors in translation and rotation. This experiment illustrates how the pIC is as robust and precise as methods designed to have good performance under these conditions, and it is better than the standard ICP. Furthermore, the pIC converges faster than previous methods. Although we have tried to give the maximum generality with the scans selected, the conclusions given are valid for these scans. To confirm these results we present next an experiment with real motion in a real scenario.

The second experiment corresponds to a run in our University with the wheelchair vehicle. The robot traveled 70 meters getting out of an office, traveling around a corridor and coming back to the office. The experiment is difficult because the floor was very polished and the vehicle slipped constantly with a poor effect on the odometry. In addition, there were glass in several parts of the run providing a significant number of spurious measurements and the corridor was quite long with not many frontal structure to correct the location in this direction.

Figure 6 depicts the results obtained with the IDC and the pIC methods. We see how the visual result of the pIC is better than the IDC, since it is able to align the corridor and the office when it comes back. The rotational accumulated error is lower for the pIC than for the IDC. Moreover, note how the error in translation is also quite small. In this experiment the scans changed from one iteration to another (involving issues as spurious and new parts of the scenario). The mean convergence rate was 23 for the pIC and 33 for the IDC. Thus, these experiments show how under more realistic conditions the behaviour of the pIC is 


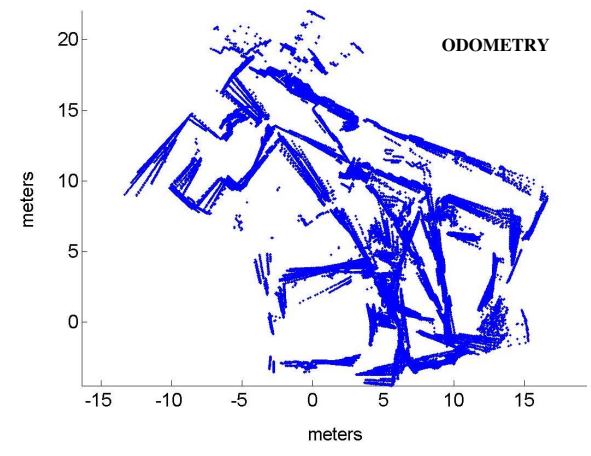

a

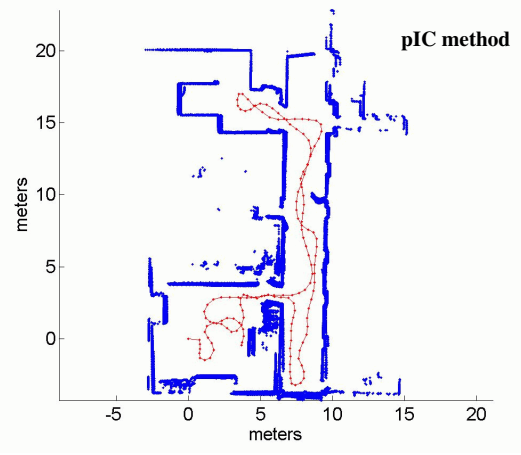

b

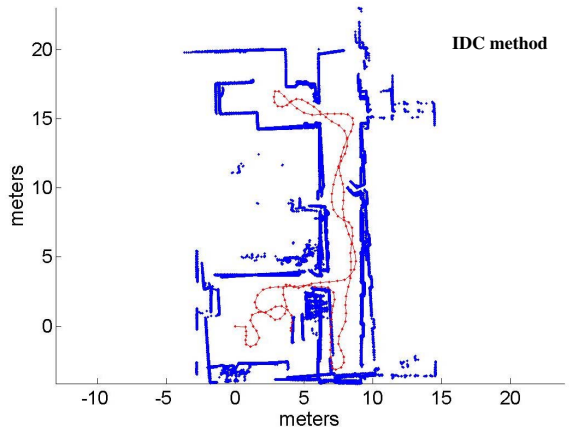

c

Fig. 6. Laser data and vehicle trajectory obtained with (a) raw odometry, (b) pIC method and (c) IDC method.

globally better than in the IDC (robustness, accuracy and convergence).

\section{CONCLUSIONS}

This paper presents a probabilistic scan matching algorithm to estimate the robot planar displacement by matching dense two-dimensional range scans. The contribution is a probabilistic modelling of this process that takes into account the uncertainty of the sensor location and the noises of the measurement process. This increases the robustness of the method in real scenarios with respect to pure geometrical methods.

Furthermore this framework captures the translation and rotation simultaneously. As a consequence our method is able to deal with large odometry errors especially in rotation, which is the difficulty of most of the existing approaches and has deserved a lot of discussion in this discipline.

We have implemented and tested the technique in a real vehicle and compared with the Iterative Dual Correspondece scan matching (IDC) algorithm and the standard ICP. The results demonstrate that we improve previous methods in accuracy, robustness and convergence.

\section{ACKNOWLEDGMENTS}

We thank José Neira for fruitful discussions and comments. This work was partially supported by MCYT DPI2003-7986.

\section{REFERENCES}

[1] O. Bengtsson and A.-J. Baerveldt. Localization by matching of range scans - certain or uncertain? In EURobot'01, Lund, Sweden, 2001.

[2] P. Besl and N. McKay. A method for registration of 3-d shapes. IEEE Transactions on Pattern Analysis and Machine Intelligence, 14:239-256, 1992.

[3] P. Biber and W. Strafler. The normal distributions transform: A new approach to laser scan matching. In IEEE Int. Conf. on Intelligent Robots and Systems, Las Vegas, USA, 2003.

[4] D. Cheverikov, D. Svirko, and P. Krsek. The trimmed iterative closest point algorithm. In International Conference on Pattern Recognition, volume 3, pages 545-548, 2002.

[5] I. Cox. Blanche: An experiment in guidance and navigation of an autonomous robot vehicle. IEEE Transactions on Robotics and Automation, 7:193-204, 1991.
[6] J. Gonzalez and R. Gutierrez. Direct motion estimation from a range scan sequence. Journal of Robotics Systems, 16(2):73-80, 1999.

[7] A. Grossmann and R. Poli. Robust mobile robot localization from sparse and noisy proximetry readings using hough transform and probability grids. Robotics and Autonomous Systems, 37:1-18, 2001.

[8] J.-S. Gutmann and K. Konolige. Incremental mapping of large cyclic environments. In Conference on Intelligent Robots and Applications (CIRA), Monterey, CA, 1999.

[9] J.-S. Gutmann and C. Schlegel. Amos: Comparison of scan matching approaches for self-localization in indoor environments. In 1st Euromicro Workshop on Advanced Mobile Robots, 1996.

[10] D. Hähnel, D. Fox, W. Burgard, and S. Thrun. A highly efficient fastslam algorithm for generating cyclic maps of large-scale environments from raw laser range measurements. In IEEE/RSJ International Conference on Intelligent Robots and Systems, Las Vegas, Usa, 2003.

[11] S. Lacroix, A. Mallet, D. Bonnafous, G. Bauzil, S. Fleury, M. Herrb, and R. Chatila. Autonomous rover navigation on unknown terrains: Functions and integration. International Journal of Robotics Research, 21(10-11):917-942, Oct-Nov. 2002.

[12] Y. Liu. Improving icp with easy implementation for free form surface matching. Pattern Recognition, 37:211-226, 2004.

[13] F. Lu and E. Milios. Robot pose estimation in unknown environments by matching $2 \mathrm{~d}$ range scans. Intelligent and Robotic Systems, 18:249-275, 1997.

[14] J. Minguez, L. Montesano, and L. Montano. An architecture for sensor-based navigation in realistic dynamic and troublesome scenarios. In IEEE Int. Conf. on Intelligent Robot and Systems, Sendai, Japan, 2004.

[15] L. Montesano, J. Minguez, and L. Montano. Modeling the static and the dynamic parts of the environment to improve sensor-based navigation. In IEEE International Conference on Robotics and Automation (ICRA), 2005.

[16] L. Montesano and L. Montano. Identification of moving objects by a team of robots using kinematic information. In IEEE Int. Conf. on Intelligent Robots and Systems (IROS), pages 284-290, 2003.

[17] S. Pfister, K. Kreichbaum, S. Roumeliotis, and J. Burdick. Weighted range sensor matching algorithms for mobile robot displacement estimation. In In Proceedings of the IEEE International Conference on Robotics and Automation (ICRA), pages 1667-74, 2002.

[18] S. Rusinkiewicz and M. Levoy. Efficient variants of the icp algorithm. In International Conference 3DIM, 2001.

[19] D. Schulz, W. Burgard, D. Fox, and A. Cremers. Tracking Multiple Moving Targets with a Mobile Robot using Particle Filters and Statistical Data Association. In IEEE Int. Conf. on Robotics and Automation, Seoul, Korea, 2001.

[20] C.-C. Wang, C. Thorpe, and S. Thrun. Online simultaneous localization and mapping with detection and tracking of moving objects: Theory and results from a ground vehicle in crowded urban areas. In Proceedings of the IEEE International Conference on Robotics and Automation (ICRA), 2003.

[21] G. Weiss and E. von Puttkamer. A map based on laserscans without geometric interpretation. In Intelligent Autonomous Systems 4 (IAS4), 1995. 